\title{
Repetitive Transcranial Magnetic Stimulation-Induced Changes in Sensorimotor Coupling Parallel Improvements of Somatosensation in Humans
}

\author{
Burkhard Pleger, ${ }^{1}$ Felix Blankenburg, ${ }^{1}$ Sven Bestmann, ${ }^{1}$ Christian C. Ruff, ${ }^{1,2}$ Katja Wiech, ${ }^{1,3}$ Klaas E. Stephan, ${ }^{1}$ \\ Karl J. Friston, ${ }^{1}$ and Raymond J. Dolan ${ }^{1}$ \\ ${ }^{1}$ Wellcome Department of Imaging Neuroscience and ${ }^{2}$ Institute of Cognitive Neuroscience, Institute of Neurology, London WC1N 3BG, United Kingdom, \\ and ${ }^{3}$ Department of Human Anatomy and Genetics, Oxford University, Oxford OX1 3QX, United Kingdom
}

Repetitive transcranial magnetic stimulation (rTMS) is an established technique for non-invasive stimulation of human cortex. Although studies have shown an influence of rTMS on single cortical regions and on simple behavioral response patterns, its influences on the dynamics of task-related activity in cortical networks have not been characterized. We provide such a characterization by showing that 5 Hz rTMS over primary somatosensory cortex (SI) induces a reconfiguration of activity patterns in a sensorimotor network, comprising the stimulated region and ipsilateral primary motor cortex (MI). These plastic changes endure for up to $120 \mathrm{~min}$ and are correlated with behavioral improvement in discrimination. Dynamic causal modeling showed that this reconfiguration could be explained by an rTMSinduced increase in SI excitability (self-connection) and an increase in the effective connectivity from SI to MI. Thus, our data demonstrate that rTMS can temporarily induce behaviorally relevant reorganization within a complex cortical network underlying human somatosensory experience.

Key words: functional magnetic resonance imaging; repetitive transcranial stimulation; sensorimotor coupling; effective connectivity; plasticity; perception

\section{Introduction}

Cortical representations are not fixed entities but are modified dynamically by experience (Buonomano and Merzenich, 1998), training (Elbert et al., 1995; Classen et al., 1998), and learning (Karni et al., 1995). The underlying neurophysiological mechanisms governing these plastic changes after manipulation of inputs are increasingly well understood. Adaptive cortical changes in response to external inputs have been demonstrated in nonhuman brains with repetitive electrical stimulation of distinct brain regions that modulate the efficiency of synaptic transmission in the stimulated neuronal tissue (Herron et al., 1986; Larson and Lynch, 1986; Stanton and Sejnowski, 1989; Bliss and Collingridge, 1993). Although such stimulation protocols were unrelated to any task and also independent of attention or higher cognitive function, their application has enhanced our understanding of cortical synaptic plasticity.

In the human brain, repetitive transcranial magnetic stimulation (rTMS) allows direct stimulation of cortical brain regions in a non-invasive manner. Depending on the temporal characteristics and the intensity of the stimulation, rTMS can temporarily enhance (Post et al., 1999) or inhibit excitability (Chen et al.,

Received Sept. 27, 2005; revised Jan. 3, 2006; accepted Jan. 5, 2006.

This work was supported by a Wellcome Trust programme grant (R.J.D.). We thank Nikolaus Weiskopf and Raffael Kalisch for fruitful discussions.

Correspondence should be addressed to Burkhard Pleger, Wellcome Department of Imaging Neuroscience, Institute of Neurology, 12 Queen Square, London WC1N 3BG, UK. E-mail: b.pleger@fil.ion.ucl.ac.uk.

DOI:10.1523/JNEUROSCI.4097-05.2006

Copyright $\odot 2006$ Society for Neuroscience $\quad$ 0270-6474/06/261945-08\$15.00/0
1997) and produce controllable, consistent, long-lasting effects on cortex physiology (Huang et al., 2005). Because most recent studies focused on the effects of rTMS on human primary motor cortex (MI) (Schlaghecken et al., 2003; Kobayashi et al., 2004; Ziemann, 2004), little is known about its influences on other brain regions. For example, it has been shown recently that highfrequency $(5 \mathrm{~Hz})$ rTMS applied to human primary somatosensory cortex (SI) evokes sustained changes in cortical excitability, indexed by suppression of the normally present paired-pulse inhibition after passive median nerve stimulation (Ragert et al., 2004). Apart from evidence suggesting that rTMS can induce physiological changes in distinct regions of the cortex, it has not been established whether rTMS influences dynamic interactions in complex networks that underpin higher-level functions.

In the present study, we investigated the effect of enhanced cortical excitability by high-frequency ( $5 \mathrm{~Hz}$ ) rTMS to left SI on both evoked brain activity and behavioral performance during a tactile frequency discrimination task. This enabled us to examine the relationship between rTMS-induced behavioral and neurophysiological alterations, i.e., changes in somatosensory discrimination performance and changes in both the local activity and functional coupling of cortical regions involved in somatosensory processing.

\footnotetext{
Materials and Methods

Subjects

The study was approved by the Joint Ethics Committee of the Institute of Neurology and National Hospital for Neurology and Neurosurgery London and was performed in accordance with the Declaration of Helsinki
} 
(1964). We recruited 16 right-handed healthy subjects who gave their written informed consent. Three subjects had to be excluded after the preceding training sessions (see Fig. 1) because they did not reach a sufficient psychophysical performance beyond chance level. Because localization of human SI can vary across individuals, we assessed the position of the TMS coil during stimulation (for additional details, see section rTMS below). In three female participants, coil position was found over the superior parietal lobe [Montreal Neurological Institute (MNI) coordinates of the coil position exceeded the mean value plus twice the SD]. Thus, data of 10 subjects (six male, aged between 20 and 37 years, mean of $29 \pm 6.5$ years) were considered for group analyses of real rTMS effects. Data of the three female participants (aged between 19 and 27 years, mean of $23 \pm 4$ years) in whom we delivered real rTMS to the superior parietal lobe were used to control for the tactile sensation elicited at the scalp.

\section{Experimental procedures}

The frequency discrimination task we used was based on recent monkey studies (see Fig. 2) (Romo et al., 1999). During the continuous acquisition of brain scans, two different stimulation frequencies were applied to the subject's right index finger in a two-alternative forcechoice design. Subsequently, subjects had to indicate whether the first or the second frequency was higher. Each subject underwent the experiment twice, one time under real and one time under sham rTMS in a counterbalanced design. To exclude carryover effects, the second run was performed at least $48 \mathrm{~h}$ after the first.

Within each run (sham and real rTMS), subjects underwent an initial event-related functional magnetic resonance imaging (fMRI) session before rTMS after they had reached a stable psychophysical criterion during two preceding training sessions (Fig. 1). This pre-rTMS fMRI session was included to control for day-by-day changes in frequency discrimination acuity and cortical hemodynamics. After the pre-rTMS fMRI session, participants were taken out of the scanner, and rTMS was applied over the left SI, $2 \mathrm{~cm}$ posterior to the "hot spot" of the right first dorsal interosseous (FDI) representation on MI (Maldjian et al., 1999). Seven minutes after rTMS, the fMRI measurements were repeated, allowing for the comparison of real and sham rTMS effects on performance and neuronal responses to the same task and stimuli.

\section{Event-related fMRI}

fMRI measurements were performed with a $3 \mathrm{~T}$ head scanner (Magnetom Allegra; Siemens, Erlangen, Germany). For the acquisition of blood oxygenation level-dependent (BOLD) volumes, we used a gradient echo $\mathrm{T} 2^{*}$-weighted echo-planar imaging (EPI) sequence (echo time, $30 \mathrm{~ms}$; repetition time, $2.21 \mathrm{~s}$; flip angle, $90^{\circ}$ ). Each volume comprised 34 oblique (transversal-coronal, $-10^{\circ}$ ) slices of $2 \mathrm{~mm}$ thickness and $3 \times 3$ $\mathrm{mm}^{2}$ in-plane resolution with a slice distance of $1 \mathrm{~mm}$, which covered the whole brain excluding cerebellum. A total of 519 volumes per session (Fig. 1, s1...s3) were acquired continuously. A high-resolution anatomical image was acquired for coregistration with the functional data using an isotropic three-dimensional spoiled gradient-recalled acquisition in a steady state sequence with 107 sagittally orientated slices covering the whole brain.

\section{Frequency discrimination task}

For electrical finger stimulation, we used a Digitimer (Welwyn Garden City, UK) DS7A stimulator with disposable surface adhesive-electrodes
(Spes Medica, Battipaglia, Italy) mounted on the radial side of the right index finger, with the cathode to the distal and the anode to the proximal phalanx. Stimulation intensity was adjusted to 2.5 times the sensory threshold. Electrodes were not removed between sessions. During each fMRI session, subjects performed a two-alternative forced-choice frequency discrimination task that comprised 70 trials and 14 so-called null trials in a randomized order (Fig. 2) (Fig. 3 shows the cortical regions that are involved in the task). These null trials had the same duration as "real" trials but did not comprise tactile stimulation. This approach has been shown to be optimal for event-related studies because it maintains good efficiency for testing both categorical and differential effects (Henson, 2003). During the task, subjects were instructed to strictly fixate a little cross in the center of the presentation screen. Within each trial, electric stimuli with different frequencies were applied to the right index finger ( $\mathrm{f} 1$ and $\mathrm{f} 2$; f indicates frequency), each for $1 \mathrm{~s}$, separated by an interstimulus interval of 2-4 s (randomly jittered in steps of $1 \mathrm{~s}$ ). The range of both frequencies was between 20 and $36 \mathrm{~Hz}$, but either $\mathrm{f} 1$ or $\mathrm{f} 2$ was higher (randomized within each session, same mean frequency for all subjects). The resulting absolute difference between $\mathrm{f} 1$ and $\mathrm{f} 2$ for each trial was $1-7 \mathrm{~Hz}$.

During each session, a total of 10 trials were presented for each frequency difference. Three to $5 \mathrm{~s}$ after the application of $\mathrm{f} 2$, subjects had to decide within $2 \mathrm{~s}$ whether $\mathrm{f} 1$ or $\mathrm{f} 2$ was higher by pressing a button with the left (nonstimulated) index finger, once for $\mathrm{f} 1$ or twice for $\mathrm{f} 2$. The decision period was triggered by a lowercase " $r$ " that replaced the fixation cross on the presentation screen until the subjects pressed the button. No feedback was given. Two to $3 \mathrm{~s}$ after the decision period, $\mathrm{f} 1$ of the following trial was applied. 


\section{rTMS}

We used a MAGSTIM Rapid Stimulator (Magstim, Whitland, Dyfed, UK) connected to a figure-of-eight-shaped coil for application of rTMS. Subjects were seated in a comfortable chair. We identified the right FDI representation on MI using single-pulse TMS applied to the left hemisphere as the position in which TMS elicited highest motor-evoked potentials (MEPs). Motor threshold (MT) was measured at the relaxed FDI muscle of the right hand. It was defined as the lowest intensity capable of evoking 5 of $10 \mathrm{MEPs}$ with an amplitude of at least $50 \mu \mathrm{V}$. The position of the right index finger representation in the left SI was chosen by moving the coil $2 \mathrm{~cm}$ posterior in the parasagittal direction (Maldjian et al., 1999). The TMS output intensity was then adjusted to $90 \%$ of the MT. During the rTMS procedure, we continuously monitored activity of the right FDI muscle by surface EMG recordings to exclude a spread of excitation.

For rTMS, a total of 25 trains of pulses were applied through the tangentially oriented coil positioned over SI with the grip pointing backwards (Fig. 1). Each of these 25 trains consisted of 50 single pulses of $5 \mathrm{~Hz}$ lasting for $10 \mathrm{~s}$, with an intertrain interval of $2 \mathrm{~s}$. The 25 trains were grouped into blocks. One block consisted of five trains, resulting in a total of five blocks and 1250 TMS pulses. The application of one block was followed by a stimulus-free interval of $1 \mathrm{~min}$ before the subsequent block was applied. In the sham condition, the coil was oriented to the same position as described for the real rTMS but was tilted $90^{\circ}$ off the surface of the head, so that only the edge of the coil touched the scalp. rTMS application was well tolerated in all subjects, because no side effects were reported. Finally, the position of the TMS coil during pulse application was marked with a vitamin E capsule. After rTMS application, with the capsule still fixed over the point of stimulation, subjects underwent postrTMS fMRI (s2) and a structural MRI. The structural scan was coregistered to the first functional scan. Using Statistical Parametric Mapping (SPM), we localized the capsule and assessed its MNI coordinates. Subjects in which the MNI coordinates exceeded the mean value plus twice the SD were excluded from additional analysis. To assess the spatial relationship between the TMS coil position on the scalp and the rTMSinduced cortical changes, we calculated the Euclidean distance between the coordinates of the capsule localization and the two peak clusters (see Fig. 4, "B").

\section{Data analysis}

Functional neuroimaging. For pre-processing and statistical analysis of the fMRI data, we used the SPM software package (SPM2; Wellcome Department of Imaging Neuroscience, London, UK). The first six volumes of each session during which BOLD signal reached steady state were discarded from additional analysis. Movement artifacts of the remaining 513 volumes were removed using realignment and unwarping (Andersson et al., 2001). Subsequently, volumes were corrected for differences in image acquisition using slice-time correction. Then the volumes were spatially normalized to the standard template of the Montreal Neurological Institute (voxel size, $2 \mathrm{~mm}^{3}$ ) to establish interindividual comparability (Friston et al., 1995). Volumes were smoothed with a $10 \mathrm{~mm}$ (full-width half-maximum) isotropic, three-dimensional Gaussian filter.

At the first or within-subject level, we modeled session-specific responses with a linear convolution model. This comprises regressors that were generated by convolving the stimulus function with a hemodynamic response function. At the second level, the main effect of frequency discrimination was assessed by comparing the real events and null events across all subjects using a paired $t$ test ( $p=0.0001$, uncorrected for multiple comparisons).

To test for rTMS-induced plasticity, we assessed the interaction between session (pre vs post) and TMS (real vs sham) using a two-stage procedure. At the first level, we computed cortical responses as the estimated discrimination-related activation for each session, in each subject. We then tested for the interaction at the between-subject or second level using a simple paired $t$ test ( $p=0.001$ for peak height, uncorrected for multiple comparisons). This was implemented by subtracting the cortical responses (post - pre) under sham rTMS from the equivalent contrast under real rTMS. The ensuing contrasts reflect an rTMS-related change, appropriately adjusted for nonspecific confounds. These same data were also used in a parametric analysis to test for correlations between rTMS-induced plasticity and behavioral improvement. To improve the sensitivity of our analyses, we additionally tested for interactions in small volumes restricted to SI as defined using the cytoarchitectonic toolbox for SPM (Eickhoff et al., 2005) as assessed in a separate second-level analysis ( $p=0.05$ for peak height, corrected for multiple comparisons).

The assignment of activation clusters to cortical areas was made according to the individual structural scans coregistered to the first image of the EPI series.

Behavioral data. We tested for effect of real rTMS on frequency discrimination ability using a paired $t$ test [real rTMS(post-pre) vs sham rTMS(postpre)]. Perceptual changes were expressed by differences in the percentage of correct responses across all frequencies ( $\mathrm{fl}-\mathrm{f} 2=1-7 \mathrm{~Hz}$ ).

Correlations between behavioral and physiological measures. To assess a possible relationship between rTMS-induced BOLD signal changes and the perceptual gain in frequency discrimination, we used an additional model that tested for significant correlations between rTMS-induced fMRI signal changes in peak voxels in SI and MI and changes in the frequency discrimination accuracy. For this model, the individual contrast maps representing the rTMS effect [post-pre corrected for sham condition; real rTMS(post-pre) - sham rTMS(post-pre)] constituted the data, and the changes in the percentage of correct responses were inserted as covariate [real rTMS(post-pre) - sham rTMS(post-pre)].

Analysis of effective connectivity (dynamic causal modeling). To assess the effective connectivity between identified brain regions within SI and MI (see Fig. 4), we used an established approach to the identification of nonlinear input-state-output systems from fMRI data, dynamic causal modeling (DCM). The central idea behind DCM is to treat the brain as a deterministic nonlinear dynamic system that is subject to inputs and produces outputs. By using a bilinear approximation to the dynamics of interactions among states, the parameters of the implicit causal model reduces to three sets (Friston et al., 2003). In the present study, these comprise (1) parameters of the direct input to the system (sensory inputs to area " $A$ " in SI as described in the inset of Fig. 4), (2) parameters that represent the strengths of the connections among the regions, and (3) parameters representing the context-dependent (rTMS) modulation of the connections. Identification proceeds in a Bayesian framework given known input functions (i.e., the experimental manipulations) and the observed responses of the system (i.e., the measured BOLD time series of the areas).

For each subject, the fMRI data of the real rTMS session (pre and post) were concatenated. The design matrix contained one regressor representing the onsets of all extrinsic inputs to SI and an additional regressor that modeled the contextual change attributable to rTMS application in the post-session. In each subject, we extracted three regional time series by computing the first eigenvariate across all voxels within a sphere of 6 $\mathrm{mm}$ radius centered on the coordinates of three regions shown in the inset of Figure 4: (1) peak voxel of the frequency discrimination within SI [see Fig. 4, "A" (MNI coordinates $-54,-27,54$ ) (see also Fig. 3)] (supplemental Table 1, available at www.jneurosci.org as supplemental material); (2) peak voxel of the positive correlation analysis within SI [see Fig. 4, "C" (MNI coordinates $-39,-30,60)$ ]; and (3) peak voxel of the negative correlation analysis within MI [see Fig. 4, "D” (MNI coordinates $-45,-12,54)]$. For each subject, these time series were embedded into five alternative DCMs to estimate the individual coupling strengths of intrinsic connections and contextual variables (see Fig. 6b) (supplemental Fig. 1, available at www.jneurosci.org as supplemental material). We then used Bayesian model selection (Penny et al., 2004) to assess the different hypotheses, represented by the different models. The comparison across subjects revealed that model 1 had the highest evidence. This model was chosen for second-level analyses (group analysis); one-sample $t$ tests were used to infer that intrinsic connections and their rTMSinduced changes were $>0$.

\section{Results}

\section{Frequency discrimination task}

Subjects first practiced the frequency discrimination task in two consecutive training sessions outside the scanner (mean correct 
responses $\pm \mathrm{SE}$; first test session, $73.62 \pm$ $3.2 \%$; second test session, $72.94 \pm 2.68 \%$ ). Before rTMS application (Fig. 1, s1), frequency discrimination ability in the real and sham condition did not differ (prereal vs pre-sham; paired $t$ test, $p=0.17$ ). The motor threshold was $56.76 \pm 2.53 \%$, and the rTMS intensity was adjusted to $51.3 \pm 2.29 \%$ of the maximum output intensity. We assessed the effect of rTMS in terms of discrimination accuracy by computing the interaction between rTMS (real vs sham) and session (pre vs post). We included an additional experimental factor with seven levels of stimulus frequency. However, in this report, which focuses on real and sham rTMS, we pooled over these levels. In paired $t$ tests, we showed that real rTMS (pre, $72.2 \pm 3 \%$; post, $81.29 \pm$ $3.74 \% ; p=0.001)$, but not sham rTMS (pre, $76.43 \pm 3.99 \%$; post, $78 \pm 4.31 \%$; $p=0.51)$, significantly improved discrimination performance. The interaction between rTMS and session also reached significance (post-pre real vs post-pre sham; paired $t$ test, $p=0.003$ ).

\section{Event-related fMRI}

For fMRI data, the cortical responses to tactile discrimination were estimated by comparing trials requiring discrimination with null trials (see Materials and Methods). In line with recent studies, discrimination-related activations were observed in primary sensorimotor cortex (Recanzone et al., 1992b; Hernandez et al., 2000; de Lafuente and Romo, 2002; Harris et al., 2002) and the secondary somatosensory cortex (Francis et al., 2000; Romo et al., 2002) contralateral to the stimulated index finger. In addition, we found significant activations in the supplementary motor area (Romo et al., 1993), as well as the premotor cortex (Hernandez et al., 2002; Romo et al., 2004), anterior insula, and prefrontal cortex (Romo et al., 1999) of both hemispheres (Fig. 3) (supplemental Table 1, available at www.jneurosci.org as supplemental material).

The effects of rTMS on evoked responses to somatosensory stimulation were estimated from the interaction between rTMS and session. This analysis showed that real compared with sham rTMS led to both an enlargement and enhancement of activation in the left rTMS-stimulated SI (Fig. 4). The Euclidean distance between the TMS coil position on the scalp (mean $\pm \mathrm{SD} ; x$, $-41.1 \pm 21.8 ; y,-26.3 \pm 30.7 ; z, 90 \pm 10$ ) and the rTMSinduced cortical changes was between 30.08 and $33.75 \mathrm{~mm}$. Note that the vertical distance (distance between the $z$ coordinates) was already between 30 and $33 \mathrm{~mm}$. Thus, the rTMS-induced cortical changes were localized directly under that position on the scalp that was chosen to apply the rTMS.

Correlations between behavioral and physiological measures To examine the relationship between rTMS-induced signal changes and the perceptual gain in frequency discrimination, we performed a correlation analysis at the between-subject level. We found two clusters of rTMS-induced BOLD signal changes that correlated with the behavioral improvement in somatosensory discrimination performance: one cluster was located in SI (area $1 / 3 \mathrm{~b}$ ) and was positively correlated with the gain in frequency perception (Figs. 4, "C", $5 a$ ), whereas a second cluster was situated within MI (Figs. 4, "D", 5b) and was negatively correlated with the gain in discrimination ability. Moreover, using an additional between-subject correlation analysis, we also tested for a direct relationship between SI and MI activity. We found a negative correlation between the activations from both peak voxels (Fig. 6a), suggesting a functional interaction between SI and MI.

Analysis of effective connectivity (dynamic causal modeling) To explore the effective connectivity between SI and MI within subjects, we used DCM (Friston et al., 2003). Time series of three regions (for locations, see Fig. 4, “A," "C," "D") were modeled with five different DCMs. These DCMs were used to compare different hypotheses or models as to how the connection strengths between SI and MI subregions changed as a result of TMS application (Fig. 6b) (supplemental Fig. 1, available at www.jneurosci.org as supplemental material). We selected the DCM with the highest model evidence for group analyses (for 
a

Correlation between increase in BOLD contrast within SI (area 1/3b; $39,30,60$ ) and gain in discrimination ability

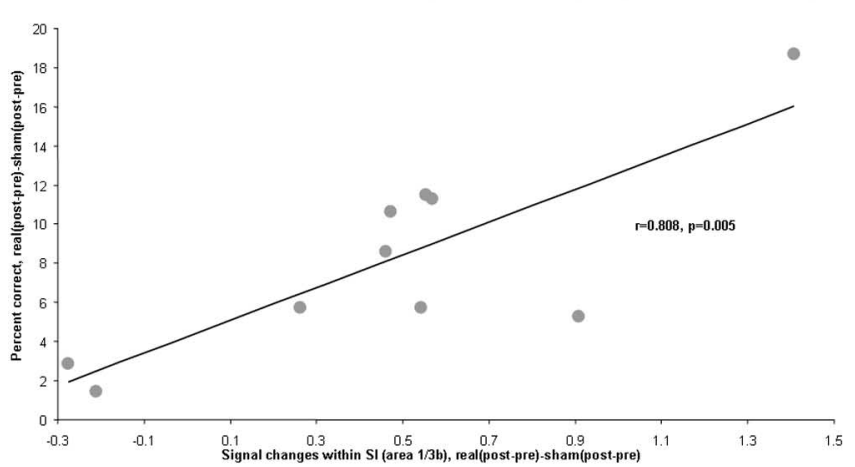

b

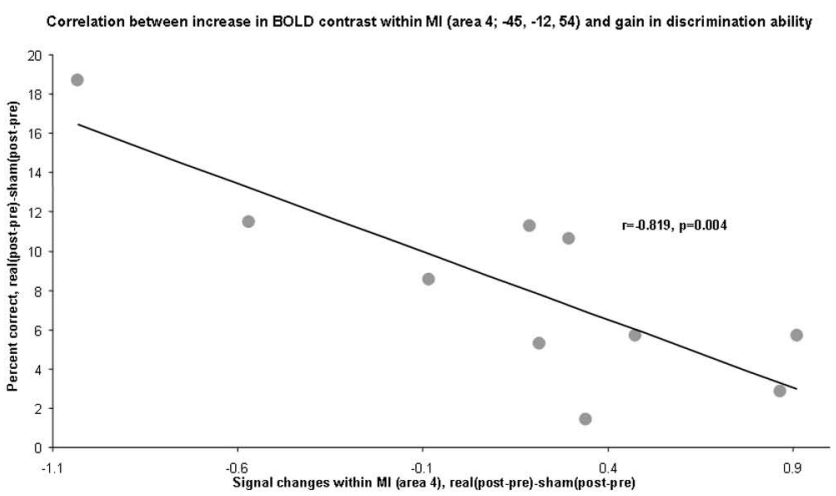

Figure 5. Correlation between cortical and psychophysical changes: $\boldsymbol{a}$, The scatter plot shows the positive correlation between signal changes in SI (area 1/3b) and the gain in discrimination abilities. $\boldsymbol{b}$, This plot shows the negative correlation between signal changes in MI (area 4a) and the psychophysical improvements. This tight relationship between cortical signal changes and perceptual gain suggests that subjects showing the highest activation gain in $\mathrm{SI}$ and the lowest activation gain in MI had the greatest behavioral improvement.

additional details, see Materials and Methods) (Penny et al., 2004). This model (Fig. 6b) involves projections within SI ("A" to " $C$ ") that propagate inputs to MI (" $C$ " and " $D$ "). The coupling strengths of the intrinsic connections were significant, emphasizing the existence of the assumed anatomical connections. Cortically, we found a significant modulatory influence of rTMS (contextual variable) only on connections within the directly stimulated SI and the connection between SI and MI. Conversely, in relation to the connections within MI, no significant modulatory effect of rTMS was found (Fig. 6b).

\section{Temporal characteristics and spatial specificity of rTMS effects}

To assess the duration of the rTMS-induced effects, three subjects were chosen randomly out of the group and were retested 120 min after termination of rTMS (Fig. 1, s3). In line with recent findings (Tegenthoff et al., 2005), cortical and psychophysical changes recovered $2 \mathrm{~h}$ after stimulation (supplemental Fig. 2, available at www.jneurosci.org as supplemental material). To test the spatial selectivity of the rTMS effects, three other subjects out of the group (randomly chosen) underwent the identical pre/post fMRI protocol while testing the index finger of the left hand with rTMS still applied over the right index finger representation. Both cortical signals and frequency discrimination ability remained unchanged, demonstrating the hemispherical specificity a

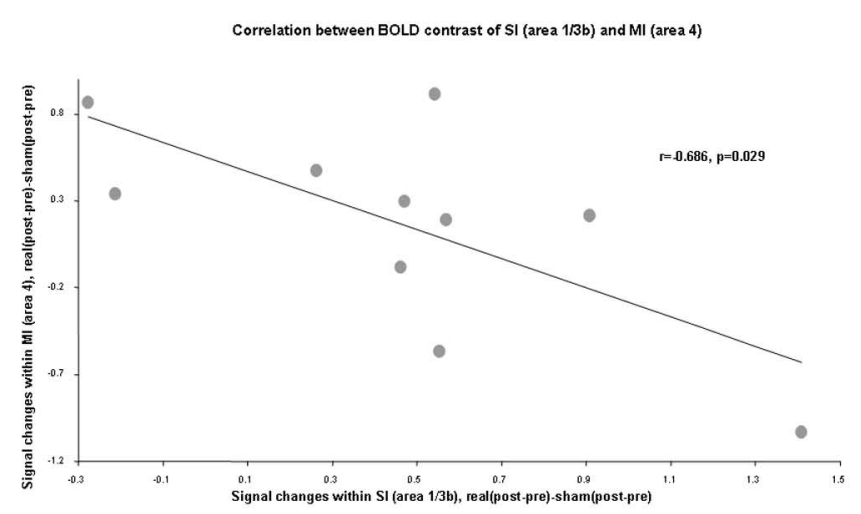

b

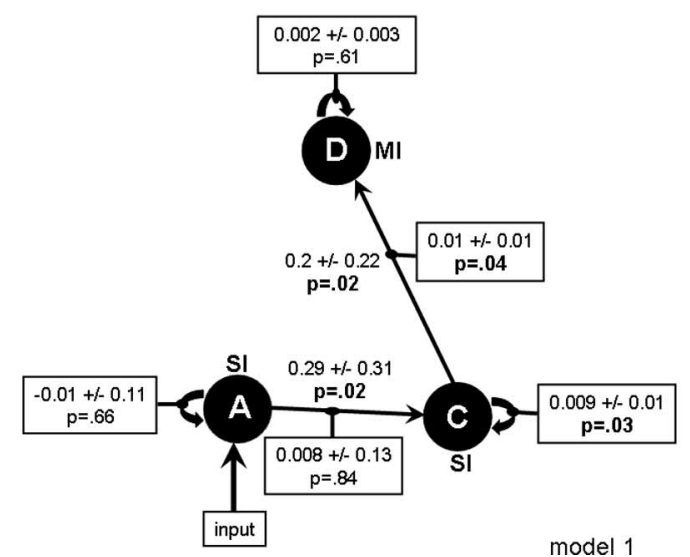

Figure 6. Sensorimotor interaction. $\boldsymbol{a}$, The correlation between psychophysical and cortical changes, based on between-subject variance, imply a functional interaction between $\mathrm{SI}$ and $\mathrm{MI}$. Indeed, testing for a correlation of BOLD signals from SI (area 1/3b) and MI (area 4a) peak voxels within subjects revealed a significant negative correlation. $\boldsymbol{b}$, DCM model 1. The letter in each circle refers to the different region as described in the inset of Figure 4. Arrows indicate the direction of coupling. The values shown represent normalized rate constants of coupling (in Hertz). The values next to the arrows represent the coupling strength of the intrinsic connections, and the values in the boxes represent the contextual (bilinear) parameters (pre vs post rTMS). $p$ values indicate results from one-sample $t$ test over subjects. Note that contextual parameters of connections within the directly stimulated SI and the connection between SI and Ml reached significance (" $C$ " and " $D$ "). Conversely, no significant modulatory effect of rTMS was found on the connections within Ml.

of the induced effects (supplemental Fig. 3, available at www. jneurosci.org as supplemental material).

\section{Control for tactile sensations elicited at the scalp}

To test whether tactile sensations elicited at the scalp contributed to the observed cortical changes, we compared perceptual accuracy and cortical signal changes before and after rTMS in three subjects in which we delivered real rTMS to the superior parietal lobe instead of the postcentral gyrus. After stimulation of the superior parietal lobe, we however found no alterations of signal changes within SI and no changes in the frequency discrimination accuracy (supplemental Fig. 4, available at www.jneurosci.org as supplemental material).

\section{Discussion}

In line with recent studies (Ragert et al., 2004; Tegenthoff et al., 2005), we found that intermittent $5 \mathrm{~Hz}$ rTMS applied to SI enhances the evoked hemodynamic signals within the directly stimulated region. These regionally specific and enduring cortical effects were negatively correlated with enhanced signals within the 
motor cortex. Changes in SI physiology and sensorimotor coupling were tightly correlated with perceptual improvements that were specific for the skin regions whose representation falls within the stimulated cortical region.

It is known that extensive training is paralleled by changes in cortical representations that speaks strongly to the relevance of cortical plasticity to experience-dependent behavioral change (Recanzone et al., 1992a; Elbert et al., 1995; Braun et al., 2001). In the somatosensory domain, changes in an individual percept can correlate with plastic changes in the associated SI representations, suggesting that the amount of cortical change attributable to changes in the peripheral input statistic is closely linked to alterations in the discrimination ability (Dinse et al., 2003; Pleger et al., 2003). In the present study, we used an artificial approach of modulating SI excitability. We applied rTMS to induce lasting changes in SI excitability, thereby shortcutting ascending somatosensory input.

The present findings suggest an enhanced SI activation after rTMS application. However, it seems also possible that rTMS induces an overall decrease in SI activation. The resulting lower background activation in SI during the post-rTMS fMRI session may in turn cause a relative increase in the response induced by the vibrotactile stimulus. Considering possible physiological consequences of rTMS, Ragert et al. (2004) asked whether the same stimulation protocol we used in the present study evokes inhibitory or facilitating changes in SI excitability. To assess excitability changes, they applied a paired-pulse protocol consisting of paired electrical stimulation of the median nerve and recorded the somatosensory-evoked potentials. After termination of rTMS, they found a sustained suppression of the normally present paired-pulse inhibition, whereas latencies and amplitudes of the first peak remained unchanged. These findings suggest a sustained enhancement and not an inhibition of SI activation.

Although the applied rTMS stimulation contains no behavioral or contextual information per se, we found that the amount of rTMS-induced response gain in SI correlated positively with the perceptual improvement. Comparable observations have been made for the so-called coactivation: $3 \mathrm{~h}$ of costimulation of neighboring receptive fields on the tip of the index finger in humans revealed its substantial potential to alter tactile performance in parallel to cortical enlargement (Dinse et al., 2003; Pleger et al., 2003). The application of both protocols, coactivation as a peripheral and rTMS as a central stimulation, has shown that unattended manipulations of the input statistics alone are sufficient to drive cortical and perceptual changes when they fulfill the requirement of high-frequency repetition and simultaneity (Tegenthoff et al., 2005).

In contrast to the positive correlation between SI signal changes and the perceptual gain, enhanced signal strength within the more frontally located primary motor cortex was negatively correlated with the improvement in frequency discrimination. In other words, subjects showing the highest activation gain in SI and the lowest activation gain in MI had the greatest behavioral improvement. These findings, based on between-subject variance, imply a functional interaction between primary somatosensory and motor cortices. Indeed, testing for a correlation of BOLD signals from SI and MI peak voxels within subjects revealed a significant negative correlation.

On testing for induced changes in effective connectivity between those regions, we found that only connections within SI and between SI and MI were modulated by rTMS. Conversely, no significant modulatory effect of rTMS was found in relation to the connections within MI. Overall, these findings of behavioralphysiological correlations and effective connectivity changes suggest a task-related dynamic reconfiguration of large-scale neural networks that is a consequence of rTMS application to SI. It should be noted that integration between sensory-attentional and motor-intentional systems is important for responding to, or orienting to, environmental stimuli. One feature of the primary somatosensory and motor cortex is the complex network of fibers that link its functional subregions (Porter, 1997). Our knowledge of the specific relationships between neurons that form these pathways is limited. In mammals, it is known that cutaneous input modulates the firing rate of motor cortical cells (Lemon, 1981). In particular, each of the functionally specialized subdivisions within SI provides the motor cortex with distinct integrated signals and contributes to motor behavior (Porter, 1991). In humans, passive cutaneous finger stimulation (Maertens de Noordhout et al., 1992; Ridding and Rothwell, 1999; Kobayashi et al., 2003) and many tactile discrimination tasks (Liu et al., 1999; Harada et al., 2004) evoke rapid adjustment of motor cortex activity based on incoming sensory information. This dynamic reconfiguration of motor circuits, as a consequence of altered peripheral inputs, strongly supports the existence of afferent sensorimotor projections in humans. Our findings go one step further in showing that behaviorally independent stimulation to selected cortical regions can result in local signal increases and perceptual improvement within the corresponding cutaneous representational territory. This artificially enhanced input to SI influences motor areas, presumably through afferent connections, a process that might reflect a graded motor preparation (Ohara et al., 2001).

Although the rTMS-induced cortical changes were localized directly under the position on the scalp that was chosen to apply rTMS, a direct influence of rTMS on MI cannot be ruled out. Two of our findings, however, support the view that the effects were mediated vicariously through changes in SI and an increased excitability in SI to MI inputs. First, rTMS-induced signal changes were found only within SI and not MI. Second, we found a modulatory role of rTMS on connections within the stimulated SI and SI to MI projections but found no direct influence of rTMS on MI.

Recent studies combining psychophysical and neurophysiological measurements in behaving monkeys have provided new insights into how different cortical areas interact to solve a vibrotactile discrimination task (de Lafuente and Romo, 2002; Hernandez et al., 2002; Romo et al., 1993, 1999, 2002, 2004). As in the present study, monkeys discriminated the difference in frequency between two periodic mechanical vibrations applied sequentially to the fingertips. The periodic mechanical vibrations can be represented in both the periodicity and the firing rate responses across the quickly adapting neuronal population within SI (Hernandez et al., 2000). SI, in turn, drives higher cortical areas in which past and current sensory information are combined, such that a comparison of the two evolves into a behavioral decision (Romo and Salinas, 2003). Although we found discriminationrelated activations in the same cortical areas, rTMS-related changes were found only within SI. The amount of rTMSinduced response gain in SI correlated positively with the improvement in frequency discrimination. Overall, these findings suggest that, although integration of several cortical areas is needed to solve the task, an improvement in tactile acuity may rely on activation changes only within SI.

The temporary reconfiguration of the stimulated SI lasted no longer than $120 \mathrm{~min}$. Zhou and Fuster (1996) trained monkeys to perform a haptic delayed matching-to-sample task with objects 
of identical dimensions but different surface features. These authors showed that, during the memory retention or delay period of the task, the activity of a cell within the SI hand area can be characterized by changes in firing frequency (Zhou and Fuster, 1996) and firing pattern (Bodner et al., 2005). This suggests that cells in SI participate not only in the perception but in the shortterm memory of tactile stimuli. In our study, the lack of significant signal changes in other regions known to contribute to sensory working memory (Burton and Sinclair, 2000) suggests that, also in humans, SI mediates not only an on-line processing but may also contribute to transient storage of sensory inputs (Harris et al., 2002). Because the parallel perceptual improvement correlated with signal changes in both SI and MI, it appears that changes in discrimination performance are closely related to induce changes in sensorimotor coupling. Although the TMS pulses applied contain no behavioral or contextual characteristics, in the context of a discrimination task, they lead to a meaningful reorganization in sensorimotor coupling that is expressed also in an improvement of perceptual thresholds for the associated skin regions.

\section{References}

Andersson JL, Hutton C, Ashburner J, Turner R, Friston K (2001) Modeling geometric deformations in EPI time series. NeuroImage 13:903-919.

Bliss TV, Collingridge GL (1993) A synaptic model of memory: long-term potentiation in the hippocampus. Nature 361:31-39.

Bodner M, Shafi M, Zhou YD, Fuster JM (2005) Patterned firing of parietal cells in a haptic working memory task. Eur J Neurosci 21:2538-2546.

Braun C, Heinz U, Schweizer R, Wiech K, Birbaumer N, Topka H (2001) Dynamic organization of the somatosensory cortex induced by motor activity. Brain 124:2259-2267.

Buonomano DV, Merzenich MM (1998) Cortical plasticity: from synapses to maps. Annu Rev Neurosci 21:149-186.

Burton H, Sinclair RJ (2000) Attending to and remembering tactile stimuli: a review of brain imaging data and single-neuron responses. J Clin Neurophysiol 17:575-591.

Chen R, Classen J, Gerloff C, Celnik P, Wassermann EM, Hallett M, Cohen LG (1997) Depression of motor cortex excitability by low-frequency transcranial magnetic stimulation. Neurology 48:1398-1403.

Classen J, Liepert J, Wise SP, Hallett M, Cohen LG (1998) Rapid plasticity of human cortical movement representation induced by practice. J Neurophysiol 79:1117-1123.

de Lafuente V, Romo R (2002) A hidden sensory function for motor cortex. Neuron 36:785-786.

Dinse HR, Ragert P, Pleger B, Schwenkreis P, Tegenthoff M (2003) Pharmacological modulation of perceptual learning and associated cortical reorganization. Science 301:91-94.

Eickhoff SB, Stephan KE, Mohlberg H, Grefkes C, Fink GR, Amunts K, Zilles K (2005) A new SPM toolbox for combining probabilistic cytoarchitectonic maps and functional imaging data. NeuroImage 25:1325-1335.

Elbert T, Pantev C, Wienbruch C, Rockstroh B, Taub E (1995) Increased use of the left hand in string players associated with increased cortical representation of the fingers. Science 220:21-23.

Francis ST, Kelly EF, Bowtell R, Dunseath WJ, Folger SE, McGlone F (2000) fMRI of the responses to vibratory stimulation of digit tips. NeuroImage 11:188-202.

Friston KJ, Ashburner J, Frith CD (1995) A spatial registration and normalization of images. Hum Brain Mapp 3:165-189.

Friston KJ, Harrison L, Penny W (2003) Dynamic causal modelling. NeuroImage 19:1273-1302.

Harada T, Saito DN, Kashikura K, Sato T, Yonekura Y, Honda M, Sadato N (2004) Asymmetrical neural substrates of tactile discrimination in humans: a functional magnetic resonance imaging study. J Neurosci 24:7524-7530.

Harris JA, Miniussi C, Harris IM, Diamond ME (2002) Transient storage of a tactile memory trace in primary somatosensory cortex. J Neurosci 22:8720-8725.

Henson RNA (2003) Analysis of fMRI time series. In: Human brain function, Ed 2 (Frackowiak RSJ, Friston KJ, Frith C, Dolan RJ, Price CJ, Zeki S, Ashburner J, Penny WD, eds), pp 793-822. San Diego: Academic.
Hernandez A, Zainos A, Romo R (2000) Neuronal correlates of sensory discrimination in the somatosensory cortex. Proc Natl Acad Sci USA 97:6191-6196.

Hernandez A, Zainos A, Romo R (2002) Temporal evolution of a decisionmaking process in medial premotor cortex. Neuron 33:959-972.

Herron CE, Lester RA, Coan EJ, Collingridge GL (1986) Frequencydependent involvement of NMDA receptors in the hippocampus: a novel synaptic mechanism. Nature 322:265-268.

Huang YZ, Edwards MJ, Rounis E, Bhatia KP, Rothwell JC (2005) Theta burst stimulation of the human motor cortex. Neuron 45:201-206.

Karni A, Meyer G, Jezzard P, Adams MM, Turner R, Ungerleider LG (1995) Functional MRI evidence for adult motor cortex plasticity during motor skill learning. Nature 377:155-158.

Kobayashi M, Ng J, Theoret H, Pascual-Leone A (2003) Modulation of intracortical neuronal circuits in human hand motor area by digit stimulation. Exp Brain Res 149:1-8.

Kobayashi M, Hutchinson S, Theoret H, Schlaug G, Pascual-Leone A (2004) Repetitive TMS of the motor cortex improves ipsilateral sequential simple finger movements. Neurology 62:91-98.

Larson J, Lynch G (1986) Induction of synaptic potentiation in hippocampus by patterned stimulation involves two events. Science 232:985-988.

Lemon RN (1981) Functional properties of monkey motor cortex neurones receiving afferent input from the hand and fingers. J Physiol (Lond) 311:497-519.

Liu Y, Gao JH, Liotti M, Pu Y, Fox PT (1999) Temporal dissociation of parallel processing in the human subcortical outputs. Nature 400:364-367.

Maertens de Noordhout A, Rothwell JC, Day BL, Dressler D, Nakashima K, Thompson PD, Marsden CD (1992) Effect of digital nerve stimuli on responses to electrical or magnetic stimulation of the human brain. J Physiol (Lond) 447:535-548.

Maldjian JA, Gottschalk A, Patel RS, Detre JA, Alsop DC (1999) The sensory somatotopic map of the human hand demonstrated at 4 Tesla. NeuroImage 10:55-62.

Ohara S, Mima T, Baba K, Ikeda A, Kunieda T, Matsumoto R, Yamamoto J, Matsuhashi M, Nagamine T, Hirasawa K, Hori T, Mihara T, Hashimoto N, Salenius S, Shibasaki H (2001) Increased synchronization of cortical oscillatory activities between human supplementary motor and primary sensorimotor areas during voluntary movements. J Neurosci 21:9377-9386.

Penny WD, Stephan KE, Mechelli A, Friston KJ (2004) Comparing dynamic causal models. NeuroImage 22:1157-1172.

Pleger B, Foerster A-F, Ragert P, Dinse HR, Schwenkreis P, Malin JP, Nicolas V, Tegenthoff M (2003) Functional imaging of perceptual learning in human primary and secondary somatosensory cortex. Neuron 40:643-653.

Porter LL (1991) Patterns of connectivity in the cat sensory-motor cortex: a light and electron microscope analysis of the projection arising from area 3a. J Comp Neurol 312:404-414.

Porter LL (1997) Morphological characterization of a cortico-cortical relay in the cat sensorimotor cortex. Cereb Cortex 7:100-109.

Post RM, Kimbrell TA, McCann UD, Dunn RT, Osuch EA, Speer AM, Weiss SR (1999) Repetitive transcranial magnetic stimulation as a neuropsychiatric tool: present status and future potential. J ECT 15:39-59.

Ragert P, Becker M, Tegenthoff M, Pleger B, Dinse HR (2004) Sustained increase of somatosensory cortex excitability by $5 \mathrm{~Hz}$ repetitive transcranial magnetic stimulation studied by paired median nerve stimulation in humans. Neurosci Lett 356:91-94.

Recanzone GH, Jenkins WM, Hradek GT, Merzenich MM (1992a) Progressive improvement in discriminative abilities in adult owl monkeys performing a tactile frequency discrimination task. J Neurophysiol 67:1015-1030.

Recanzone GH, Merzenich MM, Jenkins WM, Grajski KA, Dinse HR (1992b) Topographic reorganization of the hand representation in cortical area $3 \mathrm{~b}$ owl monkeys trained in a frequency-discrimination task. J Neurophysiol 67:1031-1056.

Ridding MC, Rothwell JC (1999) Afferent input and cortical organisation: a study with magnetic stimulation. Exp Brain Res 126:536-544.

Romo R, Salinas E (2003) Flutter discrimination: neural codes, perception, memory and decision making. Nat Rev Neurosci 4:203-218.

Romo R, Ruiz S, Crespo P, Zainos A, Merchant H (1993) Representation of 
tactile signals in primate supplementary motor area. J Neurophysiol 70:2690-2694.

Romo R, Brody CD, Hernandez A, Lemus L (1999) Neuronal correlates of parametric working memory in the prefrontal cortex. Nature 399:470-473.

Romo R, Hernandez A, Zainos A, Lemus L, Brody CD (2002) Neuronal correlates of decision-making in secondary somatosensory cortex. Nat Neurosci 5:1217-1225.

Romo R, Hernandez A, Zainos A (2004) Neuronal correlates of a perceptual decision in ventral premotor cortex. Neuron 41:165-173.

Schlaghecken F, Munchau A, Bloem BR, Rothwell J, Eimer M (2003) Slow frequency repetitive transcranial magnetic stimulation affects reaction times, but not priming effects, in a masked prime task. Clin Neurophysiol 114:1272-1277.

Stanton PK, Sejnowski TJ (1989) Associative long-term depression in the hippocampus induced by hebbian covariance. Nature 339:215-218.

Tegenthoff M, Ragert P, Pleger B, Schwenkreis P, Foerster AF, Nicolas V, Dinse HR (2005) Improvement of tactile discrimination performance and enlargement of cortical somatosensory maps after $5 \mathrm{~Hz}$ rTMS. PLoS Biol 3:e362.

Zhou YD, Fuster JM (1996) Mnemonic neuronal activity in somatosensory cortex. Proc Natl Acad Sci USA 93:10533-10537.

Ziemann U (2004) TMS induced plasticity in human cortex. Rev Neurosci 15:253-266. 